\title{
Immunotherapy - 2076. A controlled study of delta inulin-adjuvanted honey bee venom immunotherapy
}

\author{
Robert Heddle ${ }^{1 *}$, Paul Russo ${ }^{2}$, Nikolai Petrovsky ${ }^{3}$, Rory Hanna ${ }^{4}$, Anthony Smith ${ }^{5}$ \\ From 2nd WAO International Scientific Conference (WISC 2012) \\ Hyderabad, India. 6-9 December 2012
}

\section{Background}

Honey bee (HB) venom immunotherapy (HBVIT) reduces the frequency of immediate generalised reactions (IGR) to subsequent sting by only $~ 80 \%$ and in the process induces IGR in many subjects. In influenza vaccine studies delta inulin adjuvant enhanced immunogenicity and provided antigen-sparing without any adverse reactions. In an ongoing double-blinded clinical trial we are studying the benefits of inulin adjuvant for HBVIT.

\section{Methods}

Following institutional ethics committee approval, 26 subjects with a history of IGR to HB sting were randomized 2:1 to receive Albey (Stallergenes) HBVIT (100 mcg maintenance) by clustered, semi-rush regime with (gp A) or without (gp B) Advax ${ }^{\mathrm{TM}}$ inulin adjuvant (Vaxine Pty Ltd). Specific IgE (sIgE) was measured by CAP, and specific IgG1 and IgG4 by ELISA.

\section{Results}

Clinicians remain blinded to patient randomization. Two subjects have withdrawn for personal reasons. One subject had two anaphylactic reactions at 3 and 100ug of venom, was withdrawn and on breaking code was in gp B (no adjuvant). Two other subjects had mild systemic reactions. A major difference between groups is apparent in sIgG4 responses. Both groups showed a peak sIgG4 response at 14 weeks (early maintenance HBVIT). In gp A however, the sIgG4 rise started earlier, the peak response was much higher and after 12 months of maintenance HBVIT, sIgG4 levels were $\sim 3$ fold higher by ELISA OD [results mean (SEM); baseline gp A 0.110 (0.032),gp B 0.076 (0.038), peak .gp A $.0 .822(0.155)$, gp B $0.326((0.106), 52$ weeks gp A 0.453 (0.223) gp B 0.170 (0.059)]. sIgE responses showed a wide scatter with a rise from baseline of similar magnitude but occurring earlier in group $\mathrm{A}$, followed by a progressive fall, [baseline gp A 0.960 ((0.171), gp B 0.624 (0.138), peak gp A. $1.242(0.154)$, gp B $0.888(0.163)$, 52 weeks gp A $0.862(0.243)$, gp B $0.541(0.117)]$. sIgG1 responses showed a similar pattern.

\section{Conclusions}

With the caveat that only surrogate markers have yet been analysed, delta inulin adjuvant appears to enhance the immunogenicity of HBVIT and to favour sIgG4 responses.

\begin{abstract}
Author details
${ }^{1}$ Royal Adelaide Hospital, Flinders University, Adelaide. ${ }^{2}$ Royal Adelaide Hospital, Australia. ${ }^{3}$ Flinders University, Vaxine Pty Ltd, Australia. ${ }^{4}$ Royal Adelaide Hospital, Australia. ${ }^{5}$ Flinders Medical Centre, Australia.
\end{abstract}

Published: 23 April 2013

doi:10.1186/1939-4551-6-S1-P158

Cite this article as: Heddle et al:: Immunotherapy - 2076. A controlled study of delta inulin-adjuvanted honey bee venom immunotherapy. World Allergy Organization Journal 2013 6(Suppl 1):P158. 